\title{
APORTES A LA TEORIA DEL DISEÑO DESDE UNA UISIÓN EPISTÉMICA: EL GIRO SOCIAL Y SU INFLUENCIA EN UNA NUEUA AGENDA PARA LA DISCIPLINA
}

\section{Carlos Eduardo BURGOS}

Director del Instituto Interdisciplinario de Investigación en Diseño FAU - UNNE. Profesor titular ordinario de Teoría del Diseño I y II Carrera de Arquitectura; profesor de Metodología de la Ciencia Aplicada al Diseño, carreras de Arquitectura y Diseño Gráfico, FAU-UNNE.

Email: carloseb@arq.unne.edu.ar; carloseduardo.burgos@gmail.com

Palabras Clave: Diseño tecnológico, cambio social, disciplina.

Keywords: Technological design, social change, discipline.

\section{RESUMEN}

La teoría del diseño ha renovado radicalmente sus escenarios en los últimos cincuenta años. Desde el énfasis en la creación de objetos ligados al mercado, hasta los procesos de innovación social que componen la agenda actual. Presento, desde la metodología del análisis crítico y la sistematización teórica, los principales argumentos epistémicos de este giro social sobre la actividad, mostrando el desplazamiento de una impronta profesionalista hacia otra disciplinarista de mayor complejidad e integración. Concluyo con una visión del diseño más vinculada con la ética y con la democracia actual, conforme con nuevos valores y roles en el escenario cultural.

\section{ABSTRACT}

Design theory has radically renewed its stages in the last fifty years. From the emphasis in the creation of objects linked to the market, up to the processes of social innovation that compose the current agenda items. I present here, from the methodology of critical analysis and theoretical systematization, the main arguments of this epistemic shift on social activity, showing the displacement of a profesionalista stamp to a disciplinarista stamp, with more complexity and integration. I conclude with a design vision more related to ethics and $\mathrm{Cu}$ rrent democracy, according to new values and roles in the cultural scene. 


\section{OBJETIVO}

Analizar el cambio de objeto y de rol operados en el diseño tecnológico, que va desde una lógica centrada en la producción de objetos (con énfasis en la forma y la utilidad) a una lógica centrada en el cambio y la innovación social (con énfasis en el bienestar, el desarrollo y la preservación del ambiente).

\section{INTRODUCCIÓN}

Existen modelos conceptuales que intentan vincular el desafío del contexto actual sobre el territorio del diseño y los procesos de evolución de la disciplina en los últimos cincuenta años a través de sus diferentes objetos, metodologías y finalidades (WASSERMAN, 2011; DILNOT, FRIEDMAN ET ÁL. 2005; PENIN, 2006; BUCHANAN, 1985; MARGOLIN, 1989). La evolución del pensamiento proyectual (design thinking) muestra los cambios que se operaron en el interior de la disciplina, centrada primero en la característica de los artefactos (artifact-centric) y, luego, en los métodos formales de producción.

En una primera etapa (1950-1970) los procesos están signados por la influencia del mercado como una instancia privilegiada de intercambio, socialización e impacto de los productos diseñados, sobre todo en el campo del diseño industrial.

En un segundo momento, entre los 70 y los 90 , el énfasis se expresa en la interacción o interface entre el objeto y el usuario (human-centric). El hombre -como sujeto con necesidades, disposiciones perceptuales y maneras de actuar en el contextocobra relevancia especial para guiar los procesos de producción proyectual y para infundir en los productos aquellas cualidades que hacen más amigable su uso, apropiación y consumo (affordances). Como tercera etapa en la evolución, entre los 90 y hasta nuestros días, el pensamiento en diseño fue

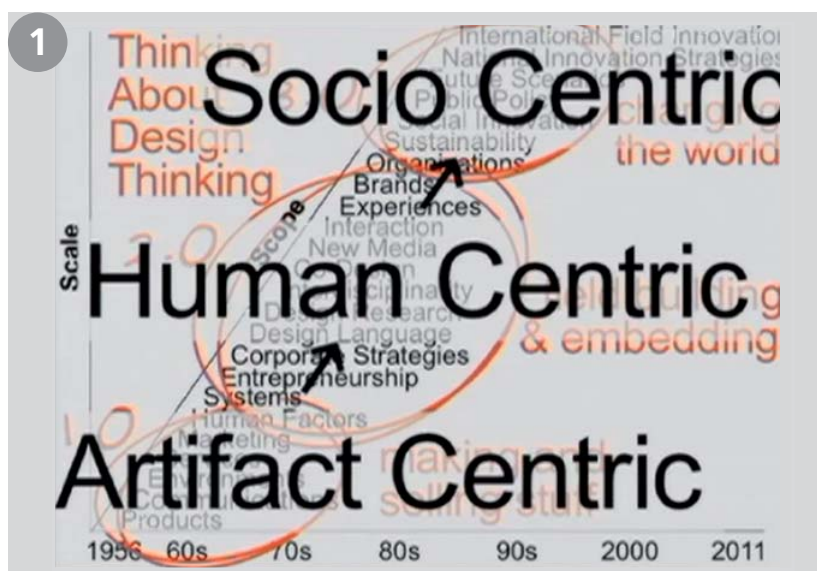

considerando con mayor atención el tratamiento de los problemas y necesidades sociales, vinculadas con el mejoramiento de la calidad de vida, el interés por el desarrollo sostenible y las demandas de los colectivos sociales en situaciones de riesgo (socio-centric).

El objetivo principal ya no es el objeto-en-sí, con sus determinaciones formales o su funcionamiento utilitario, ni tampoco la atención a las dimensiones comunicacionales de los productos, para ser más dóciles al proceso de interacción humana. Se ubican en el primer plano los procesos proyectuales orientados a la innovación y al cambio social. La consigna es ahora el diseño puede cambiar el mundo. No ya como un mero productor de objetos que se incorporan al escenario material de la cultura, sino como un motor de cambios profundos en los sistemas de producción, pensamiento y gestión de las dinámicas sociales.

Presento un esquema resumen de estas ideas, con base en dos ejes de referencias, escala y tiempo:

(En el esquema de la figura 1, se observa la evolución del proceso de pensamiento proyectual, que se inicia en los aspectos centrados en el objeto, su posibilidad objetiva, y su rol en la incorporación a los mercados. Como segunda instancia se plantea el análisis de los diferentes lenguajes, sistemas y métodos que permiten acercar al usuario (lo subjetivo). Finalmente, se presenta al diseño como un factor clave en el desarrollo político, social y cultural, basado en la innovación aplicada al cambio y la mejora social).

Este modelo tiene la virtud de poner en el escenario diferentes rasgos, improntas y centraciones del diseño en los últimos cincuenta años. Sin embargo, se plantea un esquema de etapas, en las que las "conquistas" de las instancias anteriores quedan superadas por las siguientes. Quizás sea conveniente incorporar un perfil dialéctico a este planteo histórico, en el cual los componentes comparten una misma historia, vinculados por algún rasgo común que los articula. 


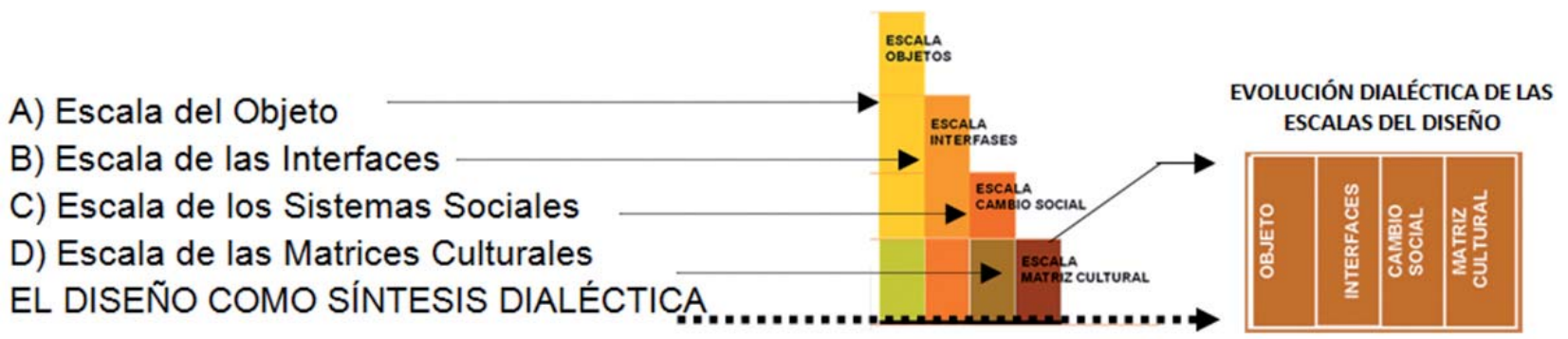

Por ello voy a proponer un modelo dialéctico, en el que las etapas van subsumiéndose en las siguientes, quedando suprimidas, aunque conservadas y superadas por la nueva escala de trabajo. Gráficamente. (Figura 2).

El esquema muestra que la disciplina no abandona los objetos de etapas anteriores, sino que los conserva dentro de su naturaleza constitutiva, donde el objeto (formal-funcional), las interfaces y sistemas de interacción (sujeto-objeto), las innovaciones en el plano social y las estructuras culturales forman parte del mismo fenómeno proyectual. En lo que sigue, presento un análisis de la escala de las innovaciones sociales, a partir de los cambios en el escenario cultural y la nueva agenda que se plantea para el diseño en la actualidad.

La escala del cambio social: el diseño como medio de transformación, innovación y bienestar social Superada la escala vinculada con el objeto, sus posibilidades e influencias en la determinación de experiencias de usuarios y los procesos relacionados con el impacto en las dinámicas de los mercados, presento aquí el nivel de análisis que vincula al diseño con otros objetivos de mayor escala, en la que se juegan necesidades y requerimientos de mayor alcance social y ambiental.

Denominaré a este fenómeno el giro social en los estudios sobre el diseño y en los roles y responsabilidades de los diseñadores. Las contradicciones entre profesión y disciplina, la confianza en la dimensión individual de los procesos metodológicos y los planteos deterministas consistentes con el clásico problem solving persisten en este nivel, que indaga en una nueva agenda social para el diseño. ${ }^{1}$

[1] El diseño ingresa en este nuevo escenario a partir de la Declaración de Ahmedabad en India, en 1979, donde el National Institute of Design discute sobre la importancia y el impacto que tiene el diseño industrial en el desarrollo de las naciones. A partir de los '70, un proceso de redefinición del campo y rol del diseño fue concebido. Quizás haya surgido en el contexto de la discusión centro/periferia o países centrales y países del Tercer Mundo. De todas maneras, ha sido un giro importante al involucrar al diseño y a los modelos de producción industrial. El eje estaría puesto, a partir de ahora, en replantear el alcance del diseño para el desarrollo de los países de una manera más efectiva, y la construcción de una estrategia más eficiente
Nuevos impactos a escala cultural. Diseño para el consumo versus diseño para la realidad: nuevas lógicas, ideas y contextos para la profesión Luego de las consecuencias devastadoras de las guerras mundiales, se tomó conciencia de la necesidad de cuidar el planeta y los recursos naturales, mejorar la calidad de vida a escala mundial y abordar cuestiones geopolíticas como la pobreza, la salud, el bienestar social y la sostenibilidad. ${ }^{2}$ Estos desafíos impregnaron también el campo del diseño, reconociendo que los seres humanos podríamos constituirnos como eje de la nueva problemática, en el ingreso al siglo XXI (FRY, 2008; MANZINI, 2009; MARGOLIN, 2007).

El diseño ha sido uno de los motores de las estructuras de producción industrial, del mercado y del consumo, alimentando los sistemas de vida a partir de la continua generación de nuevos artefactos vinculados con la idea del bienestar o confort. El diseño queda indudablemente unido a los objetos y a la idea de posesión, consumo y poder. Poder que otorga el acceso a ciertos artefactos que prolongan (técnicamente) nuestras capacidades y expectativas en nuestro proceso de relación y adaptación con el mundo. Sin embargo, esta relación de posesión y poder también insinúa su contraria, en la cual se manifiesta un marcado contraste derivado del no acceso a las ventajas del sistema de producción técnicamente industrializado y políticamente organizado. Esta relación entre consumo, acceso, poder y propiedad es también una relación de inclusión/exclusión social, ya que expone brechas importantes entre los que son poseedores $y$

de transferencia cientifico-tecnológica (MARGOLIN, 2007. 115). Para muchos autores, esta actividad ha logrado un gran impacto desde la fusión del diseño industrial con los procesos sistemáticos de la producción y, consecuentemente, por los impactos que produce sobre el mercado. En este sentido, el diseño está inexorablemente unido a la industria, sobre todo por los procesos de innovación y desarrollo conquistados en todo el siglo XX, que se han ramificado hacia fenómenos económicos, comerciales, científicos y tecnológicos de la vida social (BONSIEPE, 1991: 242).

[2] Se gesta, sí, un cambio de enfoque sobre el diseño, orientado por la idea de innovación vinculada con el bienestar. En la visión clásica, el diseño es concebido como una actividad artística y práctica (un arte liberal), que produce artefactos, muebles y productos, "this is how it is generally presented by the media and the museums. One reason why there is not more support for social design services is the lack of research to demonstrate what a designer can contribute to human welfare" (MARGOLIN, MARGOLIN, 2002: 28). 
"que el propósito más importante del diseñador hoy es asegurar el bienestar de los usuarios y del planeta" (JARAMILLO, 2011).

los que no lo son. Por ello, un nuevo lema plantea que, aunque los diseñadores pudiéramos haber contribuido al deterioro del planeta, ahora podemos aportar a su preservación, siempre que seamos capaces de proponer nuevos valores y modelos de identidad que no se fundamenten solo en las reglas del mercado y el consumo. Asumir "que el propósito más importante del diseñador hoy es asegurar el bienestar de los usuarios y del planeta" (JARAMILLO, 2011: 132), requiere la coordinación de diferentes entramados culturales y representa un trabajo de difícil realización. Ahora bien, ¿cómo puede una tarea tan trascendente y compleja ser conducida por la labor sectorial y experta de los diseñadores? Aunque le adjudiquemos un rol relevante para la producción de nuevos escenarios vitales o artificiales, no deja de ser un componente aislado de todo el complejo sistema de la construcción colectiva de la cultura actual.

Estos desafíos del escenario cultural de fines del siglo XX generaron un cambio hacia el abordaje de nuevos objetos de diseño y, también, la modificación de las aportaciones teórico-filosóficas sobre la actividad. Es posible advertir tres contextos: (i) el contexto del objeto material, concreto, dotado de capacidades funcionales y estéticas, coherente con los procesos de reproducción industrial y dócil a las lógicas del mercado; (ii) el contexto de las interfaces, que otorga una mayor atención a las experiencias de los usuarios a través de las affordances del artefacto y de la interacción entre objetos y usuarios. La producción de artefactos vinculados con el confort ha alimentado la lógica de la sociedad de consumo y considerado al diseño como un proceso apropiado para sostenerlo. (iii) El tercer contexto está dado por la focalización de los problemas sociales, no ya restringidos a los límites de los objetos-artefactos individuales, sino a cuestiones de impacto cultural y global (MORELLI, 2007).

Advierto, a partir del concepto de nuevas lógicas aplicado por MORELLI, que cada escala promueve la definición de un tipo particular de lógica, que subyace como estructura del pensamiento proyectual. ${ }^{3}$ Estas nuevas lógicas y contextos plenos de requerimientos y desafíos anclados en el seno de las problemáticas actuales derivaron en una transformación radical de la visión clásica, gestándose un giro social en los estudios y prácticas sobre el diseño. PAPANEK (1985) ofreció un replanteo de la función de los diseñadores en este nuevo marco socio-cultural, vinculado con el abordaje de las necesidades reales de las personas y sus niveles de vida, en armonía con el cuidado del medio ambiente y el rescate de los valores locales de cada comunidad. El planteo de PAPANEK significó un giro radical en el perfil de la profesión, hasta ese momento más preocupado por la definición y calidad de los objetos del diseño que por el ámbito en el que ese producto se insertaba. La contribución más importante fue exponer un escenario marcado por dos dimensiones polares: (i) el diseño "controlado" por las lógicas del mercado y la producción industrial, con la innovación vinculada con las ventas y los intereses económicos, o (ii) el diseño centrado en las políticas públicas, los principios de responsabilidad ética sobre la profesión y el compromiso social de la innovación proyectual. Este planteamiento desplaza del centro de la escena los valores vinculados con la estética y la función de los artefactos y posiciona como centrales a los procesos y productos que el diseño puede aportar para promover mejores condiciones de vida, situadas en contextos ambientales sostenibles. La foca-

[3 ] Las lógicas del objeto están dadas por su entramado formal y funcional, las de las interfaces por la relación e interacción entre diferentes propiedades y disposiciones de diferentes componentes. En este caso, la escala social promueve una lógica del empoderamiento de las capacidades individuales en el abordaje de sus propios problemas. El giro social es un desplazamiento inadvertido de los poderes creacionistas de los diseñadores — y de la competitividad económica — hacia el protagonismo de la praxis protagónica de los sujetos en sus contextos de vida. Aquí el diseño re-configura ese contenido "trivial" que se ha mencionado en el inicio y por el cual todos pasamos a ser diseñadores, es decir, protagonistas creativos de nuestro entorno. 4 Lo que PAPANEK afirma es que ha llegado la hora de abandonar los criterios de belleza y funcionalidad para re-definir al diseño y sus roles en la sociedad: "for thousands of years philosophers, artists, and designers have argued about the need for beauty and aesthetic value in the things we use and live with. Designers and engineers have insisted on optimal function. One only has to look out the window or into one's own room to see where this schizoid preoccupation with function and the look of thinks has led us: The world is ugly, but it doesn't work well either! In a world of abject want, a preoccupation with only making things pretty is a crime against humanity" (PAPANEK, 1985: 326s). 
lización exclusiva en el desarrollo de las cualidades estético-formales de los objetos y las expectativas, cada día más ambiciosas, de sus funcionamientos y performances, ha sido objeto de fuertes críticas a la hora de contrastar el valor de los productos con el mejoramiento sensible del mundo en que vivimos $y$ de las condiciones de vida que promovemos en él. En este sentido, PAPANEK ha advertido la crítica radical respecto del interés por las búsquedas estéticas y funcionalistas de los objetos, que se constituyen -en su opinión - en un crimen contra la humanidad, sobre la base de los problemas sociales y ambientales que considera más importantes. ${ }^{4}$

Es clara la crítica que marca PAPANEK sobre la impotencia de las estrategias profesionales centradas en el tratamiento de las formas y las funciones de los objetos para intervenir y transformar exitosamente nuestro mundo. Es una visión que ha sido exitosamente refutada por la realidad. Por ello, otros autores que han seguido sus huellas han encendido la alarma sobre las necesidades y deseos que -efectivamente- el diseño ha abordado, descuidando las verdaderas necesidades del hombre en este tiempo, lo que consideró como un diseño vinculado con el "mundo real", y oponiendo claramente el diseño orientado al mercado. Sus críticas se han centrado en la relativa ausencia en los programas de diseño, de las necesidades económicas, psicológicas, espirituales, tecnológicas e intelectuales del ser humano $y$, con ello, de la posibilidad para la transformación del hombre mismo en su propia cultura. El objetivo último de un diseño debe ser siempre "transformar el ambiente del hombre, sus herramientas y, por extensión, al hombre mismo" (PAPANEK, 1985: 28, traducción propia). Inevitablemente, la complejidad de la vida actual hace cada vez más difícil

[4] Lo que PAPANEK afirma es que ha llegado la hora de abandonar los criterios de belleza y funcionalidad para re-definir al diseño y sus roles en la sociedad: "for thousands of years philosophers, artists, and designers have argued about the need for beauty and aesthetic value in the things we use and live with. Designers and engineers have insisted on optimal function. One only has to look out the window or into one's own room to see where this schizoid preoccupation with function and the look of thinks has led us: The world is ugly, but it doesn't work well either! In a world of abject want, a preoccupation with only making things pretty is a crime against humanity" (PAPANEK, 1985: 326s). predecir o prescribir los cambios, aunque sea necesario orientar las estrategias a cambios posibles.

La superación de la escala del objeto:

la innovación social como nuevo modelo y objetivo del diseño tecnológico

$\mathrm{Si}$ los diseños estuvieron mayoritariamente subsumidos en la lógica del racionalismo económico, ahora, la reflexión se debería centrar en problemas de mayor alcance, como el cambio ecológico, la sustentabilidad, la habitabilidad, las condiciones de vida. MARGOLIN y BUCHANAN ven a la función social del diseño más allá de ser concebido como un productor de objetos estéticos y funcionales ligados al mercado. Desde este lugar, el diseño, "once narrowly defined as a marginal activity concerned with the aesthetic appeal of a limited range of consumer goods, can now be seen to be at the core of all our conceptions and plans for our personal and collective social lives" (BUCHANAN, MARGOLIN, 1995: 141). ${ }^{5}$

Los riesgos del diseño ligado a un modelo exclusivamente centrado en el consumo se ciernen, por un lado, sobre el ambiente y los recursos naturales, mientras que, por el otro, debilitan las condiciones protagónicas de la vida social y el acceso a recursos básicos para amplios sectores de la población mundial. Este escenario se comprende mejor en el contraste entre países centrales y periféricos, planteados desde la lógica de los negocios desarrollados por los países industrializados de Occidente. En ese aspecto, MORELLI (2007) instala la problemática de la globalización, alertando que la expansión de los mercados de los países desarrollados a otras regiones implica también una expansión de sus lógicas y

[5 ] La posición de MARGOLIN sobre el modo en que el producto nos conecta con la acción es un importante avance en la conceptualización clásica sobre el diseño. Es un buen punto de vista considerar que los objetos se transforman en estructuras que facilitan la interacción protagónica de los sujetos en el mundo (praxis). Sin embargo, quiero marcar una idea que me parece relevante: el hombre ha producido históricamente su medio y su mundo, aunque pareciera que luego de generar una porción de él (los objetos diseñados) los ve de manera extraña, como un cuerpo extraño que ingresa en el organismo, y advierte una suerte de necesidad de explorarlo, analizarlo, estudiarlo y comprenderlo como si fuera una entidad externa a sí mismo. Del marcado dualismo, aún presente en las concepciones actuales sobre el diseño, me desplazo aqui hacia un holismo genético de las estructuras culturales e históricas del hombre. 
"... Mientras que los científicos y tecnólogos focalizan sobre los aspectos físicos del metabolismo social, los diseñadores deberían concentrar sus intereses en trabajar sobre las dimensiones sociales, políticas y económicas derivadas de los procesos de la globalización (2007, p. 4)."

dinámicas de crecimiento. Desde el punto de vista del ambiente, implica catastróficas consecuencias para la preservación de los recursos naturales y una merma sensible de las condiciones de vida (p. 4).

Si el diseño se mantiene funcional a este contexto dominado por las lógicas de la competitividad y rentabilidad de las empresas y de los procesos de desarrollo de las economías de los países centrales, entonces abandona la posibilidad de producir innovaciones y cambios centrados en la mejora de las condiciones de vida de cada región. ${ }^{6}$ Es preciso que el diseño como "profesión" se asocie a otras "profesiones" para abordar estos problemas, siempre manteniendo la mirada estratégica en los contextos geopolíticos locales. Para ello, es preciso ampliar la visión restringida de la profesión clásica, sumando otras dimensiones teóricas concurrentes. Mientras que los científicos y tecnólogos focalizan sobre los aspectos físicos del metabolismo social, los diseñadores deberían concentrar sus intereses en trabajar sobre las dimensiones sociales, políticas y económicas derivadas de los procesos de la globalización (2007, p. 4).

El giro social asumido en los últimos años por teóricos, investigadores y diseñadores está produciendo una nueva visión sobre los posibles impactos que pueden generar sobre el modo de vida de las personas y sobre los sistemas sociales que se construyen en torno de los objetos diseñados. Los "problemas sociales" se transforman en un nuevo objetivo para el diseño, alterando también los métodos y los modelos epistémicos para analizar la realidad. Salta a la vista que el modelo signado por las funciones de utilidad o la estética de un objeto diseñado no es, ahora, el objetivo central. La idea de

[6 ] Esta es la posición de autores como BONSIEPE (BONSIEPE, 2006; BONSIEPE, 1985; BONSIEPE, 1991), quienes desarrollan una concepción no universalista sino estrictamente contextualizada del diseño, que debe dar cuenta — desde la periferia— de los problemas propios de la periferia. El desafío de la globalización, en este caso, deriva en la necesidad de una híper-regionalización. El compromiso y la responsabilidad dejan de ser meramente técnicos y se transforman en complejas problemáticas ideológico-políticas (CASTELLS, 2006). confort, asociada al consumo de las cosas - a veces superfluas - se torna secundaria, dejando el lugar central a aquellas vinculadas con la transformación social y el mejoramiento de las condiciones de vida. ${ }^{7}$ La antigua lógica, centrada en la búsqueda exacerbada del confort y en el consumo pasivo "disabling people, because it deprives them of the capability to solve problems in the future (...) sometimes undermines social relationships as it replaces personal links and social networks with technological products or services" (MORELLI, 2007: 6). La idea plantea la posibilidad de pérdida de protagonismo, activismo y proactividad de la sociedad en la identificación y gestión sobre sus propias problemáticas.

El giro socia/ también implica un desplazamiento de la escala de los productos a la escala de los sistemas, o complejos de relaciones que surgen a partir de los objetos y conjuntos. Se gesta la condición de posibilidad para un nuevo paradigma en la consideración de los roles del diseñador en la producción cultural, alejado de las dimensiones individuales de la práctica, habitualmente centradas en la intencionalidad del diseñador y en su capacidad metodológica ("inteligencia") especial.

Consistente con esta búsqueda y replanteo en el interior del diseño como expresión cultural (no solo profesional), se producen nuevas visiones sobre los aspectos sociales vinculados con el diseño desde una perspectiva interdisciplinaria (BUCHANAN, MARGOLIN, 1995). Los debates se iniciaron a partir de la herencia que los trabajos de autores como PAPANEK, MARGOLIN y MORELLI han dejado, constituyendo un abordaje complejo de los objetos pro-

[7 ] Es interesante el punto de vista que MORELLI expone, al plantear que este rol de consumidor confortable atenta contra una posición más activa en la construcción democrática de los espacios de vida humana. El permanente bombardeo de nuevos objetos, sistemas y dispositivos orientados al consumo conduciría a las personas hacia un estado "pasivo" en cuanto al compromiso y la responsabilidad como ciudadanos, ya que sus necesidades son definidas por un conjunto de técnicos y expertos sobre lo que, se supone, él necesita para vivir mejor. Los problemas sociales o de los grupos son representados por un conjunto de necesidades tipificables, y de su traducción a objetos de consumo depende el éxito de la función del proyecto en la cultura. 
abordar las problemáticas de la actividad, desde otros escenarios que exceden los análisis tradicionales sobre el proceso proyectual se torna provocativa..."

yectuales y con la participación de especialistas de diferentes disciplinas. ${ }^{8}$ La premisa fundamental ha sido considerar que el diseño no puede ser adecuadamente comprendido desde sus abordajes internos, con las herramientas del discurso teórico disponible desde la concepción dominante en el último tramo del siglo XX.

Por este motivo, ha sido preciso prestar atención a la acción proyectual desde otro paradigma conceptual (no-profesionalista), tanto en sus aspectos estructurales como en sus dinámicas.

La intención de re-descubrir el diseño a partir de nuevos enfoques y perspectivas - no profesionalistas - "reflects a growing recognition that the design of the every world deserves attention not only as a professional practice but as a subject of social, cultural, and philosophic investigation" (BUCHANAN, MARGOLIN, 1995: ix).

Esta manera de abordar las problemáticas de la actividad, desde otros escenarios que exceden los análisis tradicionales sobre el proceso proyectual se torna provocativa, ya que descentra el fundamento clásico que defiende la posición dominante, al pretender elucidar los rasgos esenciales de la actividad investigando la dinámica interna del ejercicio profesional, aunque exponiendo — dada su condición emergente- tensiones y contradicciones que se desprenden de sus planteamientos.

Poner a la dimensión de lo social en primer plano implica, necesariamente, advertir los problemas, conflictos y desafíos que la sociedad afronta, en parte derivados de los procesos de producción tecnológica y sus impactos sobre nuestra cultura oc-

[8] Este hecho marca la aceptación implícita de que el diseño no puede ser visto desde la óptica restringida de una mera práctica profesional, sino como una disciplina que intenta validar los contextos constitutivos de sus conocimientos, procesos, objetos e impactos. Desde este punto de vista, las tramas disciplinares de diseño se articulan e interactúan con otras tramas disciplinares (epistémicas, éticas, tecnológicas) configurando entidades cognitivas complejas, que superan en alto grado los límites de la clásica visión profesiona de la actividad. De allí es posible obtener una auto-validación, no desde la autonomía de las prácticas profesionales en sentido clásico. cidental. Es claro que el diseño no puede negar el rol que le cabe en el engranaje funcional de los procesos de producción y transformación material del mundo.

La duda que estos nuevos escenarios plantean es la siguiente: ¿se ha orientado el diseño hacia las necesidades, demandas y propósitos genuinamente humanos, o más bien hacia los mecanismos de la producción de objetos materiales ligados a intereses cercanos a las lógicas e ideologías del mercado industrial? La ecuación que se define entre los términos diseño + industria + mercado + economía = cultura tecnológica dominante es todavía un tema de debate clave en el momento de replantear el rol del diseño en la actualidad. ${ }^{9}$

Es preciso, por ello, sumar a las miradas clásicas sobre el diseño (como profesión) otras que parten desde otros contextos no restringidos al sistema de prácticas, sino que se centran en una dimensión colectiva (no individual), que permite entender mejor las tramas genéticas de los procesos vinculados con el diseño, y que condicionan las dinámicas de innovación e impacto de sus productos.

El giro socia/ también ha cambiado el sujeto destinatario del diseño. Este sujeto ahora es colectivo, ya no individual o polar (cliente-consumidor), incluso ni siquiera es un grupo de personas bajo el denominador común de ciertos intereses o rasgos de clase, sino que son entramados de diferentes actores y actantes sociales (LATOUR, 2006), que co-producen los sistemas proyectuales, a partir del propio reconocimiento de sus problemáticas y desafíos. Las competencias proyectuales se encuentran en estas redes, no son ya propiedades exclusivas de los pro-

[9] Estos temas son abordados por los estudios en ciencia, tecnología y sociedad (CTS) desde hace tiempo, llevando a replanteos importantes en las concepciones sobre la ciencia, la tecnología y sus complejos procesos de interacción con la sociedad. Lo que propongo -implíitamente — es sumar el fenómeno de las producciones e innovaciones proyectuales a este escenario, dado que tendría un efecto enriquecedor, tanto para los estudios CTS como para un abordaje más comprensivo y complejo del diseño tecnológico. 
yectistas profesionales, operando una transferencia de capacidades de diseño hacia los contextos sociales 10

El cambio de paradigma se desplaza desde los objetos hacia los sistemas, las plataformas, las estructuras y los escenarios de interacción.

En este sentido, ¿qué tipos de problemas, actividades y metodologías es posible advertir?, ¿qué nuevos roles emergen como imperativos para los diseñadores? Estas cuestiones parecieran ser los nuevos desafíos para la transformación de la disciplina en el siglo XXI y, con ella, del rol del diseñador en este nuevo escenario.

Por otra parte, desde una búsqueda de la transformación de los procesos de innovación social en los nuevos contextos de industrialización de la cultura, MORELLI (2007) destaca el contraste entre aquellos diseñadores basados en las lógicas del mercado y aquellos que comenzaron a focalizar nuevos objetos y temas de trabajo, orientados a generar contribuciones específicas para la solución de problemas vinculados con lo social o con lo ambiental.

El cambio necesario, tanto para PAPANEK como para MORELLI y otros autores (MARGOLIN, MARGOLIN, 2002, 1995, 2007; FRY, 2008; PENIN, 2006; TONKINWISE, 2004; (APRA, 2004), se condensa en los conceptos de innovación, cambio social y sostenibilidad en la producción de nuevos artefactos y sistemas técnicos.

Transformar las prácticas y los objetos sobre los cuales se trabaja implica un cambio radical en la tradición de la profesión, no ya centrada en la especificación restringida de un objeto particular (ligado al consumo), sino en la creación de nuevos sistemas e interfaces que puedan ser capaces de instalarse en los escenarios problemáticos de los diferentes contextos culturales (pobreza, marginalidad, deterioro ambiental, etc.), y permitir el protagonismo de los grupos sociales en la gestión de sus propios procesos de solución. Veremos, incluso, que el diseñador perderá en este contexto su figura protagónica como gestor metodológico principal, dejando lugar a nuevas entidades proyectantes - no necesariamente humanas-, que interactuarán en las tramas de la producción artificial de los artefactos.

\section{CONCLUSIÓN}

El análisis sobre la evolución de los objetos, valores y dinámicas proyectuales en los últimos cincuenta años genera como resultado una imagen de creciente complejidad para el fenómeno proyectual, asociado a un contexto de interacción de agencias diversas, transdisciplinares e intersectoriales. EI giro social en los estudios sobre la actividad pone en evidencia, además, la mutación de las clásicas concepciones profesionalistas en nuevas determinaciones (transdisciplinares), con un mayor protagonismo de los ámbitos socioculturales en la definición de las tramas y redes proyectuales, delineando una ontología reticular en contraste con el realismo epistémico que ha infundido las aportaciones teóricas del fenómeno proyectual hasta nuestros días.

[10 ] La profesión pierde sus rasgos individuales: el sujeto-diseñador, el objeto-producto, la función de utilidad, los estándares estéticos, el cliente-consumidor, las necesidades derivadas de la demanda, el impacto sobre el medio. Mientras el diseñador aún se debate si este conjunto de expectativas sigue definiendo su profesión, los procesos de la globalización asociados a los cambios tecnológicos y productivos han transformado radicalmente el escenario de los procesos proyectuales. El diseño en este nuevo contexto industrial corporativo debe reflexionar sobre los cambios en sus objetos de trabajo, sus metodologías sus productos $y$ dinámicas (MARGOLIN, MARGOLIN, 2002; MORELLI, 2007). 


\section{REFERENCIAS BIBLIOGRÁFICAS}

BATESON, G. (1997). Espíritu y naturaleza. Buenos Aires: Amorrortu Editores.

BECK, U. and REY, J. A. (2002). La sociedad del riesgo global. Madrid: Siglo Veintiuno.

BONSIEPE, G. (2006). Design and democracy. Design Issues, 22(2), pp. 27-34.

BONSIEPE, G. (1991). Developing countries: awareness of design and the peripheral condition, history of Indian Design: 1919-1990 the dominion of design. Milan: Electa.

BONSIEPE, G. (1985). El diseño de la periferia. Barcelona: Gustavo Gili.

BORGMANN, A. (1995). The depth of design. In: BUCHANAN, R. \& MARGOLIN, V. (Eds.) Discovering design. The University of chicago Press, pp. $13-22$. BRONCANO, F. (2005). La agencia técnica. Revista iberoamericana de ciencia tecnología y sociedad, 2(5), pp. 95-107.

BUCHANAN, R. (2001). Design and the new rhetoric: Productive arts in the philosophy of culture. Philosophy and Rhetoric, 34(3), pp. 183-206.

BUCHANAN, R. (1985). Declaration by design: Rhetoric, argument, and demonstration in design practice. Design Issues, 2(1), pp. 4-22.

BUCHANAN, R. and MARGOLIN, V. (1995). Discovering design: explorations in design studies. Chicago: University of Chicago Press.

CALLON, M. (2001). Redes tecno-económicas e irreversibilidad. Redes, 8(17), pp. 85-126.

CAPRA, F. (2004). The hidden connections: A science for sustainable living. New York: Random House Digital, Inc.

CASTELLS, M. (2006). La sociedad red: una visión global. Madrid: Alianza Editorial.

CORAGGIO, J. L. (2004). La gente o el capital: desarrollo local y economía del trabajo. Buenos Aires: Editorial Abya Yala.

CROSS, N. (2007). Designerly Ways of Knowing. Berlin: Birkhäuser Verlag AG.

CROSS, N. (1995). Discovering design ability. Chicago: University of Chicago Press.

DILNOT, C., FRIEDMAN, D., MARGOLIN, V. and TIGERMAN, S. (2005). Ethics? Design? The Archeworks Papers, 1(2), pp. 15-53.

DOUGLAS, M. (1980). Estilos de pensar. Barcelona: Gedisa.

EISENMAN, P. (2006). The formal basis of modern architecture. Cambridge: Trinity College.

FRY, T. (2008). Design futuring: Sustainability, ethics and new practice. New York: Berg Publishers.

HACKING, I. (1999). The social construction of what? Cambridge: Harvard University Press.

HOUKES, W., VERMAAS, P. E., DORST, K. and DE VRIES, M. J. (2002). Design and use as plans: an action-theoretical account. Design Studies, 23(3), pp. 303-320.

HUTCHINS, E. and LINTERN, G. (1995). Cognition in the Wild. Cambridge: MIT Press.

IBARRA, A. (2012). Epistemic networks. New subjects for new forms of (scientific) knowledge production. Science, Technology \& Innovation Studies, 8(1), pp. 61-74

IBARRA,A.(2006). Réplica a Minhot. Individual vs. colectivo y régimen epistémico. El mundo que la ciencia construye. Córdoba:Editorial Brujas, pp. 61-66. JARAMILLO, J. R. (2011). Diseñadores para nuevas ideas de bienestar, 2011. Buenos Aires: Actas Universidad de Palermo-Argentina.

KOOLHAAS, R., KOOLHAAS, R. and KOOLHAAS, R. (1994). Delirious New York: a retroactive manifesto for Manhattan. New York: Monacelli Press. LADRIĖRE, J. (1978). El reto de la racionalidad: la ciencia y la tecnología frente a las culturas. Paris: Sígueme - UNESCO.

LATOUR, B. (2006). Reassembling the Social. Política y Sociedad, 43(3), pp. 127-130.

LAWSON, B. and DORST, K. (2009). Design Expertice. Oxford: Elsevier- Architectural Press.

LÓPEZ CEREZO, J. A. and LUJÁN, J. L. (2000). Ciencia y política del riesgo. Madrid: Alianza.

MALDONADO, T. (1972). Design, nature, and revolution: toward a critical ecology. New York: Harper \& Row.

MANZINI, E. (2009). New design knowledge. Design Studies, 30 (1), pp. 4-12.

MARGOLIN, V. (2007). Design for development: towards a history. Design Studies, 28(2), pp. 111-115.

MARGOLIN, V. (2007). Design, the future and the human spirit. Design Issues, 23(3), pp. 4-15.

MARGOLIN, V. (1995). The politics of the artificial. Leonardo, 28, pp. 349-356.

MARGOLIN, V. (1989). Design discourse: history, theory, criticism. Chicago: University of Chicago Press.

MARGOLIN, V. and MARGOLIN, S. (2002). A "social model" of design: issues of practice and research. Design issues, 18(4), pp. 24-30.

MITCHAM, C. (1994). Thinking through technology: The path between engineering and philosophy. Chicago: University of Chicago Press.

MORELLI, N. (2007). Social innovation and new industrial contexts: Can designers "industrialize" socially responsible solutions? Design Issues, 23(4), pp. 3-21. 
PAPANEK, V. (1985). Design for the real world: Human ecology and social change. London: Thames and Hudson.

PENIN, L. (2006). Strategic design for sustainable social innovation in emerging contexts: framework and operative strategies. CMU School of Design 2012/2013 Lecture Series: <http.www. design.cmu.edu/designthefuture/laura-penin/>.

QUINTANILLA, M. Á. (2005). Tecnología: un enfoque filosófico. México: Fondo de Cultura Económica.

SIMON, H. (1996). The Sciences of the Artificial. 3rd ed edn. Cambridge: The MIT Press.

TONKINWISE, C. (2004)-last update, Ethics by design, or the ethos of things. Available: http://desphilosophy.com/dpp/dpp/_journal/back/paper4_ tonkinEthi.cs/dpp_paper4.html [10/21/09].

TXAPARTEGI, E. (2009). ¿Sujeto-red o sujeto-programa? En: F. BRONCANO and A. (PÉREZ-RANSANS, eds., Los sujetos de la ciencia. Madrid: Siglo XXI. WASSERMAN, A. (2011). Thinking About 50 Years of Design Thinking. CMU School of Design 2012/2013 Lecture Series edn. http://www.design. cmu.edu/designthefuture/arnold-wasserman/: Carnegie Mellon Design.

ZACCAI, G. (1995). Art and technology, aesthetics redefined. En: R. BUCHANAN and V. MARGOLIN, Eds., Discovering design: Explorations in design studies. Chicago: University of Chicago Press, pp. 3-12. 